\title{
Stochastic optimization methods in engineering
}

\author{
Kurt Marti \\ Federal Armed Forces University Munich \\ Aero-Space Engineering and Technology \\ D-85577 Neubiberg/Munich, Germany. \\ Tel: +49-89-6004-2541/2109. \\ Fax: +49-89-6004-3560. \\ e-mail: Kurt.Marti@rz . unibw-muenchen .de
}

\begin{abstract}
Yield stresses, allowable stresses, moment capacities (plastic moments), external loadings, manufacturing errors are not given fixed quantities in practice, but have to be modelled as random variables with a certain joint probability distribution. Hence, problems from limit (collapse) load analysis or plastic analysis and from plastic and elastic design of structures are treated in the framework of stochastic optimization. Using especially reliabilityoriented optimization methods, the behavioral constraints are quantified by means of the corresponding probability $p_{s}$ of survival. Lower bounds for $p_{s}$ are obtained by selecting certain redundants in the vector of internal forces; moreover, upper bounds for $p_{s}$ are constructed by considering a pair of dual linear programs for the optimizational representation of the yield or safety conditions. Whereas $p_{s}$ can be computed e.g. by sampling methods or by asymptotic expansion techniques based on Laplace integral representations of certain multiple integrals, efficient techniques for the computation of the sensitivities (of various orders) of $p_{s}$ with respect to input or design variables have yet to be developed. Hence several new techniques are suggested for the numerical computation of derivatives of $p_{s}$.
\end{abstract}

Keywords

Stochastic optimization, structural design, structural analysis, probability functions 


\section{LIMIT (COLLAPSE) LOAD ANALYSIS OF STRUCTURES AS A LINEAR PROGRAMMING PROBLEM}

The collapse load can be defined [4] "as the load required to generate enough number of local plastic yield points (referred as plastic hinges for bending type members) to cause the structure to become a mechanism and develop excessive deflections". Assuming that the material behaves as an elastic-perfectly plastic material [5], a conservative estimate of the collapse load factor $\lambda_{\mathrm{T}}$ is based on the following formulation as a linear program (LP) :

maximize $\lambda$

s.t.

$$
\begin{aligned}
& \mathrm{F}^{\mathrm{L}} \leq \mathrm{F} \leq \mathrm{F}^{\mathrm{U}} \\
& \mathrm{CF}=\lambda \mathrm{R}_{\mathrm{o}} .
\end{aligned}
$$

Here, (1.2) is the equilibrium equation of a statically indeterminate loaded structure involving an $m \times n$ matrix $c=\left(c_{i j}\right), m<n$, of given coefficients $c_{i j}$, $1 \leq i \leq m, 1 \leq j \leq n$, depending on the undeformed geometry of the structure having $n_{0}$ members (elements); we suppose that rankC $=m$.

In case of a truss [5] we have $n=n_{0}$, and the matrix $C$ contains the direction cosines of the members. Furthermore, $R_{0}$ is an external load m-vector, and $F$ denotes the $n$-vector of internal forces and bending-moments in the relevant points (sections, nodes or elements) of the structure; in case of a truss $F$ involves only the member forces. Finally, (1.1) are the yield conditions with the vector of lower and upper bounds $F^{L}, F^{U}$. For a truss we have that

$$
F_{j}^{L}=\sigma_{y j}^{L} A_{j}, F_{j}^{U}=\sigma_{y j}^{U} A_{j}, j=1, \ldots, n\left(-n_{o}\right),
$$

where $A_{j}$ is the (given) cross-sectional area, and $\sigma_{y j}^{L}, \sigma_{y j}^{U}$, resp., denotes the yield stress in compression (negative values) and tension of the $j$-th member of the truss. In case of a plane frame $F$ is composed of subvectors

$$
F^{(k)}=\left(\begin{array}{c}
F_{1}^{(k)} \\
F_{2}^{(k)} \\
F_{3}^{(k)}
\end{array}\right)
$$

where $F_{1}^{(k)}$ denotes the normal (axial) force, and $F_{2}^{(k)}, F_{3}^{(k)}$ are the bending-moments at the two ends of the $k$-th member. In this case $F^{L}, F^{U}$ contain - for each member $k$ - the subvectors

$$
F^{(k) L}=\left(\begin{array}{c}
\sigma_{y k}^{L}{ }_{y k} \\
-M_{p \ell, k} \\
-M_{p \ell, k}
\end{array}\right) \quad F^{(k) U}=\left(\begin{array}{c}
\sigma_{y, k}^{U}{ }^{A} \\
M_{p \ell, k} \\
M_{p \ell, k}
\end{array}\right)
$$


resp, , where $M_{p \ell, k}, k=1, \ldots, n_{0}$, denote the plastic moments (moment capacities) given $[4],[5]$ by

$$
M_{p \ell, k}=\sigma_{y k}^{U} W_{p \ell, k}
$$

and $W_{p l, k}=W_{p l, k}\left(A_{k}\right)$ is the plastic modulus of the cross-section of the $k$-th member (beam).

\section{PLASTIC AND ELASTIC DESIGN OF STRUCTURES}

In the plastic design of trusses, frames [5] having $n_{0}$ members, the $n$-vectors $F^{\mathrm{L}}, F^{\mathrm{U}}$ of lower and upper bounds

$$
F^{\mathrm{L}}=\mathrm{F}^{\mathrm{L}}\left(\sigma_{\mathrm{y}}^{\mathrm{L}}, \sigma_{\mathrm{y}}^{\mathrm{U}}, \mathrm{X}\right), \mathrm{F}^{\mathrm{U}}=\mathrm{F}^{\mathrm{U}}\left(\sigma_{\mathrm{y}}^{\mathrm{L}}, \sigma_{\mathrm{y}}^{\mathrm{U}}, \mathrm{X}\right)
$$

for the $n$-vector $F$ of internal member forces and bending-moments $F_{j}, j-1, \ldots$ ,$n$, are determined [3],[5] by the yield stresses, i.e. compressive limiting stresses (negative values) $\sigma_{\mathrm{y}}^{\mathrm{L}}=\left(\sigma_{\mathrm{yl}}^{\mathrm{L}}, \ldots, \sigma_{\mathrm{yn}}^{\mathrm{L}}\right)^{\prime}$, the tensile yield stresses $\sigma_{\mathrm{y}}^{\mathrm{U}}=\left(\sigma_{\mathrm{y} 1}^{\mathrm{U}}, \ldots, \sigma_{\mathrm{yn}}^{\mathrm{U}}\right)^{\prime}$, and the $\mathrm{r}$-vector

$$
x=\left(x_{1}, x_{2}, \ldots, x_{r}\right)^{\prime}
$$

of design variables of the structure. In case of trusses we simply have that, cf. (1.3),

$$
F^{L}=\sigma_{y d}^{L} A(X), \quad F^{U}=\sigma_{y d}^{U} A(X)
$$

where $\mathrm{n}^{-\mathrm{n}_{0}}$, and $\sigma_{y d}^{L}, \sigma_{y d}^{U}$ denote the $n \times n$ diagonal matrices having the diagonal elements $\sigma_{y j}^{L}$, $\sigma_{y j}^{U}, \operatorname{resp} ., j=1, \ldots, n$, moreover,

$$
A(X)=\left(A_{1}(X), \ldots, A_{n}(X)\right)^{\prime}
$$

is the $n$-vector of cross-sectional areas $A_{j}=A_{j}(X), j=1, \ldots, n$, depending on the $r$-vector $x$ of design variables $x_{k}, k=1, \ldots, r$.

Corresponding to (1.2), here we have again the equilibrium equation $\mathrm{CF}=\mathrm{R}_{\mathrm{u}}$,

where $R_{u}$ describes [5] the ultimate load (representing constant external loads or self-weight expressed in linear terms of $A(X)$ ).

The plastic design of structures can be represented then [1], [2], [5] by the following optimization problem

$\min G(X)$

s.t.

$$
\begin{aligned}
& F^{\mathrm{L}}\left(\sigma_{\mathrm{y}}^{\mathrm{L}}, \sigma_{\mathrm{y}}^{\mathrm{U}}, \mathrm{X}\right) \leq \mathrm{F} \leq \mathrm{F}^{\mathrm{U}}\left(\sigma_{\mathrm{y}}^{\mathrm{L}}, \sigma_{\mathrm{y}}^{\mathrm{U}}, \mathrm{X}\right) \\
& \mathrm{CF}=\mathrm{R}_{\mathrm{u}},
\end{aligned}
$$


where $G=G(X)$ is a certain objective function, e.g. the volume or weight of the structure.

For the elastic design we have to replace the yield stresses $\sigma_{y}^{\mathrm{L}}, \sigma_{\mathrm{y}}^{\mathrm{U}}$ by the allowable stresses $\sigma_{\mathrm{y}}^{\mathrm{L}}, \sigma_{\mathrm{y}}^{\mathrm{U}}$, and instead of ultimate loads we consider service loads $R_{s}$. Hence, instead of (3) we get the related program

min $G(X)$

s.t.

$$
\begin{gathered}
\mathrm{F}^{\mathrm{L}}\left(\sigma_{\mathrm{a}}^{\mathrm{L}}, \sigma_{\mathrm{a}}^{\mathrm{U}}, \mathrm{X}\right) \leq \mathrm{F} \leq \mathrm{F}^{\mathrm{U}}\left(\sigma_{\mathrm{a}}^{\mathrm{L}}, \sigma_{\mathrm{a}}^{\mathrm{U}}, \mathrm{X}\right) \\
\mathrm{CF}=\mathrm{R}_{\mathrm{s}} \\
\mathrm{X}^{\mathrm{L}} \leq \mathrm{X} \leq \mathrm{X}^{\mathrm{U}}, \\
\text { where } \mathrm{X}^{\mathrm{L}}, \mathrm{X}^{\mathrm{U}} \text { still denote lower and upper bounds for } \mathrm{X} \text {. }
\end{gathered}
$$

\section{ANALYSIS AND DESIGN OF STRUGTURES IN GASE OF RANDOM DATA}

In practice, yield stresses, allowable stresses, the loads applied to the structure, other material properties and the manufacturing errors are not given fixed quantities, but must be treated as random variables on a certain probability space $(\Omega, O L, P)$. Hence, (1), (3), (4) are stochastic linear/nonlinear programs (SLP/SNLP) which obviously have the same basic structure represented by a random objective function

$$
\mathrm{Z}(\omega):=\mathrm{G}(\omega, \mathrm{X}), \omega \in \Omega,
$$

and by stochastic constraints of the type

$$
\begin{aligned}
& \mathrm{CF}=\mathrm{R}(\omega) \\
& \mathrm{F}^{\mathrm{L}}(\omega) \leq \mathrm{F} \leq \mathrm{F}^{\mathrm{U}}(\omega),
\end{aligned}
$$

where $R=R(\omega), \omega \in \Omega$, is a random load $m$-vector given by

$$
R(\omega)=\lambda R_{0}(\omega), R(\omega)=R_{u}(\omega), R(\omega)=R_{s}(\omega),
$$

resp., and for the $n$-vector $F=\left(F_{j}\right)$ of internal member forces and bending-moments we have the $n$-vectors of random bounds

$$
F^{\mathrm{L}}(\omega)=F^{\mathrm{L}}(\omega, \mathrm{X}), \mathrm{F}^{\mathrm{U}}(\omega)=\mathrm{F}^{\mathrm{U}}(\omega, \mathrm{X}), \omega \in \Omega,
$$

depending on an $r$-vector $x$ of design variables $x_{k}, k=1, \ldots, r$.

Obviously, each realization of the random element $\omega \in \Omega$ yields new loading conditions, represented by the vector $R=R(\omega)$, and therefore, $c f .(6)$, new arrangements $F=F(\omega)$ of internal member forces and bending-moments. Hence, in the present case of "multiple loadings" caused by the random variations of $\mathrm{R}=\mathrm{R}(\omega)$, the survival of the structure, $i . e$. the existence of certain arrangements $F(\omega)$ of internal member forces/bending-moments not overwhelming the strength of the structure, can be evaluated by the probability of survival

$P_{\mathbf{S}}:=P($ There is $F=F(\omega)$ such that $C F(\omega)=R(\omega)$

$$
\text { and } \left.F^{L}(\omega) \leq F(\omega) \leq F^{U}(\omega)\right) \text {, }
$$

see $[2],[10]$, assuming that, cf. (26), (33),

$S\left(F^{L}(),. F^{U}().\right):=(\omega \in \Omega$ : there is a vector $F=F(\omega)$

$$
\text { fulfilling (6) and (7)) }
$$


is a measurable set. Denoting by $\left[F,{ }^{\mathrm{L}}, \mathrm{F}^{\mathrm{U}}\right]$ the $\mathrm{n}$-dimensional interval $\left[F^{\mathrm{L}}, \mathrm{F}^{\mathrm{U}}\right]:=\left(\mathrm{F} \in \mathfrak{R}^{\mathrm{n}}: \mathrm{F}^{\mathrm{L}} \leq \mathrm{F} \leq \mathrm{F}^{\mathrm{U}}\right\}$,

we find that

$S\left(F^{L}(),. F^{U}().\right)-\left\{\omega \in \Omega: R(\omega) \in C\left[F^{L}(\omega), F^{U}(\omega)\right]\right\}$

with $C\left[F^{L}, F^{U}\right]=\left\{C F: F^{L} \leq F \leq F^{U}\right\}$, and therefore

$$
\begin{aligned}
\mathrm{p}_{\mathrm{S}} & =\mathrm{P}\left(\mathrm{S}\left(\mathrm{F}^{\mathrm{L}}(.), \mathrm{F}^{\mathrm{U}}(.)\right)\right)=\mathrm{P}\left(\mathrm{R}(\omega) \in \mathrm{G}\left[\mathrm{F}^{\mathrm{L}}(\omega), \mathrm{F}^{\mathrm{U}}(\omega)\right]\right) \\
& \left.=\int \mathrm{P}\left(\mathrm{R}(\omega) \in \mathrm{C}\left[\mathrm{F}^{\mathrm{L}}, \mathrm{F}^{\mathrm{U}}\right] \mid \mathrm{F}^{\mathrm{L}}(\omega)-\mathrm{F}^{\mathrm{L}}, \mathrm{F}^{\mathrm{U}}(\omega)-\mathrm{F}^{\mathrm{U}}\right) \mathrm{P}\left(\mathrm{F}^{\mathrm{L}}(.), \mathrm{F}^{\mathrm{U}}(.)\right) \mathrm{dF}^{\mathrm{L}}, \mathrm{dF} \mathrm{F}^{\mathrm{U}}\right),
\end{aligned}
$$

where $P\left(F^{I}(),. F^{U}().\right)$ denotes the distribution of the bounds $\left(F^{\mathcal{L}}(\omega), F^{U}(\omega)\right)$.

Since the bounds $F^{\mathrm{L}}, \mathrm{F}^{\mathrm{U}}$ in (7) depend also on the vector $\mathrm{X}$ of design varia-

bles $x_{k}, k=1, \ldots, r$, cf. (7.1), we have $p_{s}=P(x)$ with the probability function

$$
P(X)=P\left(R(\omega) \in C\left[F^{L}(\omega, X), F^{U}(\omega, X)\right]\right) ;
$$

furthermore, if the external load $R=R(\omega)$ is given by

$$
R(\omega)=R(\omega, \lambda):=\sum_{i=1}^{m_{R}} \lambda_{i} R^{(i)}(\omega)
$$

with random $m$-vectors $R^{(i)}=R^{(i)}(\omega), i=1, \ldots, m_{R}$, and deterministic coefficients $\lambda_{i}, i=1, \ldots, m_{R}$, then $p_{s}=P(X, \lambda)$ with

$$
P(\lambda, X):=P\left(\sum_{i=1}^{m_{R}} \lambda_{i} R^{(i)}(\omega) \in G\left[F^{L}(\omega, X), F^{U}(\omega, X)\right]\right) .
$$

Especially, in case of trusses, see (1), (3), (4) and (2.2), for dealing with the probability of survival $\mathrm{P}_{s}$ we have the following probability functions :

$$
\begin{aligned}
P_{A}(\lambda, X): & =P\left(\lambda R_{0}(\omega) \in C\left[A(X) d_{y}^{\sigma}(\omega), A(X) d_{y}^{\sigma}(\omega)\right]\right), \lambda \in R \\
P_{u}(X): & =P\left(R_{u}(\omega) \in C\left[A(X) d_{y}^{\sigma}(\omega), A(X) d^{\sigma} \sigma_{y}^{U}(\omega)\right]\right) \\
P_{s}(X): & =P\left(R_{s}(\omega) \in C\left[A(X) d_{y}^{\sigma L}(\omega), A(X) d_{y}^{\sigma}(\omega)\right]\right),
\end{aligned}
$$

where $A_{d}=A(X)$ denotes the $n \times n$ diagonal matrix having the diagonal elements $A_{j}=A_{j}(X), j=1, \ldots, n\left(-n_{0}\right)$.

Since $p_{s}=P(X)$ are very complicated expressions in general, in the following we are looking for approximations (lower, upper bounds) of $p_{s}=P(X)$ by simpler probability functions. 
4 LOWER AND UPPER BOUNDS FOR $\mathrm{p}_{s}=\mathrm{P}(\mathrm{X})$

According to (8.3) we have that

$$
S\left(F^{L}(.), F^{U}(.)\right) \subset \bigcap_{i=1}^{m} S_{i}\left(F^{L}(.), F^{U}(.)\right)
$$

where

$$
s_{i}\left(F^{L}(.), F^{U}(.)\right):-\left\{\omega \in \Omega: R_{i}(\omega) \in C_{i}\left[F^{L}(\omega), F^{U}(\omega)\right]\right\}, i=1, \ldots, \mathbb{m},
$$

and $c_{i}$ denotes the $i-t h$ row of $c$. Hence, we get the inequality

$$
P(X) \leq \min _{1 \leq i \leq m} P_{i}(X),
$$

where

$$
P_{i}(X):=P\left(R_{i}(\omega) \in C_{i}\left[F^{L}(\omega, X), F^{U}(\omega, X)\right]\right) .
$$

Note that related inequalities (with more exact bounds) follow from more general Bonferroni-type inequalities [6]. We find that

$$
\mathrm{C}_{i}\left[\mathrm{~F}^{\mathrm{L}}(\omega, \mathrm{X}), \mathrm{F}^{\mathrm{U}}(\omega, \mathrm{X})\right]=\left[\gamma_{\mathrm{i}}^{\mathrm{L}}(\omega, \mathrm{X}), \gamma_{\mathrm{i}}^{\mathrm{U}}(\omega, \mathrm{X})\right]
$$

is an interval in $x^{1}$ having the bounds

$$
\begin{aligned}
& \gamma_{i}^{\mathrm{L}}(\omega, \mathrm{X})=\min _{1 \leq \iota \leq \mathrm{J}} \mathrm{C}_{\mathrm{i}} \mathrm{G}^{\iota}(\omega, \mathrm{X}) \\
& \boldsymbol{\gamma}_{\mathrm{i}}^{\mathrm{U}}(\omega, \mathrm{X})=\max _{1 \leq \iota \leq \mathrm{J}} \mathrm{C}_{\mathrm{i}} \mathrm{G}^{\iota}(\omega, \mathrm{X}),
\end{aligned}
$$

where $G^{\iota}=G^{\iota}(\omega, X), \iota-1, \ldots, J$, are the extreme points of the interval $\left[F^{L}(\omega, X)\right.$, $\left.F^{\mathrm{U}}(\omega, X)\right]$. Since the components $\mathbf{G}_{j}^{\iota}(\omega, X), j=1,2, \ldots, n$, of $G^{\iota}(\omega, X), \iota=1, \ldots, J$, are certain elements of $\left(F^{L}(\omega, X), F^{U}(\omega, X)\right)$, the measurability of the bounds $F^{L}, F^{U}$ with respect to $w \in(\Omega,, P)$ yields the measurability of $\gamma_{i}^{L}$ and $\gamma_{i}^{U}, i=1$, . $\ldots, m$, with respect to $w$. Hence, $s_{i}\left(F^{L}(),. F^{U}().\right), i=1, \ldots, m$, are measurable sets, and we may write

$$
P_{i}(X)=P\left(\gamma_{i}^{L}(\omega, X) \leq R_{i}(\omega) \leq \gamma_{i}^{U}(\omega, X)\right), i=1, \ldots, m .
$$

\subsection{Lower bounds by selection of redundants}

For the construction of lower bounds for $P(X)$, the vector $F=F(\omega)$ is partitioned

$$
F(\omega)=\left(\begin{array}{l}
F_{I} \\
N
\end{array}\right)
$$

into a certain $(n-m)$-vector $\mathrm{N}=\left(\mathrm{F}_{\mathrm{j}_{\ell}}\right)_{1 \leq \ell \leq n-\mathrm{m}}$ of redundants $\mathrm{F}_{j_{\ell}}, \ell=1, \ldots, \mathrm{n}-\mathrm{m}$, and an m-vector $F_{I}$ of statically determined member forces/bending-moments. Hence, with a corresponding partition of the $m \times n$ matrix $C$ into $m \times m, m \times(n-m)$ submatrices $\mathrm{C}_{I}, \mathrm{C}_{\mathrm{II}}$, resp., where

$$
\operatorname{rankC}_{I}=\operatorname{rankC}=m \text {, }
$$


the equilibrium equation (6) yields for $F(\omega)$ the representation

$$
F(\omega)=\left(\begin{array}{c}
F_{I} \\
N
\end{array}\right)=\left(\begin{array}{c}
C_{I}^{-1} R(\omega) \\
0
\end{array}\right)+\left(\begin{array}{c}
C_{I}^{-1} C_{I I} \\
I
\end{array}\right) N
$$

Consequently, selecting for each $\omega \in \Omega$ a vector of redundants

$\mathrm{N}=\mathrm{N}(\omega)=\left(\mathrm{F}_{\mathrm{j}_{\ell}}(\omega)\right)_{1 \leq \ell \leq \mathrm{n}-\mathrm{m}}$ such that $\mathrm{N}($.$) is a measurable function on (\Omega, Q, P)$,

we get

$$
\mathrm{S}\left(\mathrm{F}^{\mathrm{L}}(., \mathrm{X}), \mathrm{F}^{\mathrm{U}}(., \mathrm{X})\right) \supset \overline{\mathrm{s}}(\mathrm{X}, \mathrm{N}(.)) \text {, }
$$

where $\widetilde{S}(X, N()$.$) is the measurable set given by$

$$
\tilde{S}(X, N(.)):=\left\{\omega \in \Omega: F^{L}(\omega, X) \leq\left({ }^{C_{I}^{-1} R(\omega)-C_{I}^{-1} C_{I I} N(\omega)}\right) \leq F^{U}(\omega, X)\right\} .
$$

Thus, for $P(X)$ we find the lower bound

$$
P(X) \geq \widetilde{P}(X, N(.)) \text {, }
$$

where

$$
\tilde{\mathrm{P}}(\mathrm{X}, \mathrm{N}(.)):=\mathrm{P}\left(\begin{array}{c}
\mathrm{F}_{\mathrm{I}}^{\mathrm{L}}(\omega, \mathrm{X}) \leq \mathrm{C}_{\mathrm{I}}^{-1} \mathrm{R}(\omega)-\mathrm{C}_{\mathrm{I}}^{-1} \mathrm{C}_{I I} \mathrm{~N}(\omega) \leq \mathrm{F}_{I}^{\mathrm{U}}(\omega, \mathrm{X}) \\
\mathrm{F}_{\mathrm{II}}^{\mathrm{L}}(\omega, \mathrm{X}) \leq \mathrm{N}(\omega) \leq \mathrm{F}_{\mathrm{II}}^{\mathrm{U}}(\omega, \mathrm{X})
\end{array}\right),
$$

and $F_{I}^{L}, F_{I I}^{L}$ and $F_{I}^{U}, F_{I I}^{U}$ denotes the partition of $F^{L}, F^{U}$, resp., corresponding to (13). Note that the inequality (16) holds for any choice $N=\left(F_{j_{l}}\right)$

of an (n-m)-subvector of redundants such that (13.1) holds and any representation of $N$ as a random vector $N=N(\omega)$ on $(\Omega,, P)$; especially, $N$ can be selected as a deterministic vector of redundants:

$N(\omega)=z$ a.s. (almost sure),

where $z \in^{\mathrm{n}-\mathrm{m}}$ is a deterministic vector; in this case we set $\widetilde{\mathrm{P}}(\mathrm{X}, \mathrm{N}())=$. $\overline{\mathrm{P}}(\mathrm{X}, \mathrm{z})$.

\subsubsection{Special cases}

a) In case of trusses, cf. (1.3), (2.2), we have that

$$
\begin{aligned}
& F_{I}^{L}(\omega, X)=A_{I}(X) d^{\sigma} \sigma_{I}^{L}(\omega), F_{I}^{U}(\omega, X)=A_{I}(X){ }_{d} \sigma_{I}^{U}(\omega) \\
& F_{I I}^{L}(\omega, X)=A_{I I}(X){ }_{d}^{\sigma}{ }_{I I}^{L}(\omega), F_{I I}^{U}(\omega, X)=A_{I I}(\omega) d_{I I}^{\sigma}(\omega),
\end{aligned}
$$

where $\mathrm{A}_{\mathrm{I}}, \mathrm{A}_{\mathrm{II}}, \sigma_{\mathrm{I}}^{\mathrm{L}}, \sigma_{\mathrm{II}}^{\mathrm{L}}, \sigma_{\mathrm{I}}^{\mathrm{U}}, \sigma_{\mathrm{II}}^{\mathrm{U}}$ are the partitions of $\mathrm{A}, \sigma^{\mathrm{L}}, \sigma^{\mathrm{U}}$, resp., corresponding to the partition $F_{I}, F_{I I}$ of $F$, and $A_{I}(X)$ denotes the diagonal matrix which has the components of $A_{I}(X)$ as its diagonal elements. Thus, we get 


$$
\begin{aligned}
\widetilde{P}(X, z) & =P\left(\begin{array}{c}
A_{I}(X){ }_{d} \sigma_{I}^{L}(\omega) \leq C_{I}^{-1} R(\omega)-C_{I I}^{-1} \leq A_{I}(X){ }_{d}^{\sigma}{ }_{I}^{U}(\omega) \\
A_{I I}(X) d_{d I}^{\sigma}{ }_{I I}^{L}(\omega) \leq z \leq A_{I I}(X) d^{\sigma} \sigma_{I}^{U}(\omega)
\end{array}\right) \\
& =P\left(\begin{array}{c}
\sigma_{I}^{L}(\omega) \leq A_{I}(X) d_{d}^{-1}\left(C_{I}^{-1} R(\omega)-C_{I}^{-1} C_{I I} z\right) \leq \sigma_{I}^{U}(\omega) \\
A_{I I}(X) d^{\sigma} \sigma_{I I}^{L}(\omega) \leq z \leq A_{I I}(X) d_{I I}^{\sigma}(\omega)
\end{array}\right)
\end{aligned}
$$

b) Suppose that the partition of $F^{\mathrm{L}}, F^{U}$ into $F_{I}^{\mathrm{L}}, F_{I}^{U}$ and $F_{I I}^{L}, F_{I I}^{U}$ can be chosen such that $\left(\mathrm{F}_{\mathrm{I}}^{\mathrm{L}}(\omega, \mathrm{X}), \mathrm{F}_{\mathrm{I}}^{\mathrm{U}}(\omega, \mathrm{X})\right),\left(\mathrm{F}_{\mathrm{II}}^{\mathrm{L}}(\omega), \mathrm{F}_{\mathrm{II}}^{\mathrm{U}}(\omega)\right)$ are stochastically independent. If (17 holds, then

$$
\begin{aligned}
\tilde{P}(X, z):= & P\left(F_{I}^{L}(\omega, X) \leq C_{I}^{-1} R(\omega)-C_{I}^{-1} C_{I I} \leq F_{I}^{U}(\omega, X)\right) \\
& \times P\left(F_{I I}^{L}(\omega, X) \leq z \leq F_{I I}^{U}(\omega, X)\right) .
\end{aligned}
$$

5 FAILURE MODES, LIMIT STATE FUNCTIONS AND UPPER BOUNDS FOR $\mathrm{P}(\mathrm{X})$

According to $(8),(10)$ we have that

$P(X)=P($ There is $F=F(\omega)$ such that $C F(\omega)=R(\omega)$ and

$$
\begin{aligned}
& F_{j}(\omega)-F_{j}^{U}(\omega, X) \leq 0, j=1, \ldots, n \\
& \left.F_{j}^{L}(\omega, X)-F_{j}(\omega) \leq 0, j=1, \ldots, n\right),
\end{aligned}
$$

where we suppose first that all bounds $F_{j}^{L}, F_{j}^{U}$ are finite, i.e.

$-\infty<F_{j}^{L}(\omega, X) \leq F_{j}^{U}(\omega, X)<+\infty$ a.s., $1 \leq j \leq n$, for all $X$ under consideration.

Defining

$$
\mathrm{t}(\omega, \mathrm{F}(\omega), \mathrm{X}):=\max _{1 \leq \mathrm{j} \leq \mathrm{n}}\left\{\mathrm{F}_{\mathrm{j}}(\omega)-\mathrm{F}_{\mathrm{j}}^{\mathrm{U}}(\omega, \mathrm{X}), \mathrm{F}_{\mathrm{j}}^{\mathbf{L}}(\omega, \mathrm{X})-\mathrm{F}_{\mathrm{j}}(\omega)\right),
$$

we obtain

$P(X)=P($ There is $F=F(\omega)$ such that $C F(\omega)=R(\omega)$ and $t(\omega, F(\omega), X) \leq 0$ \}

$=P(\inf \{t(\omega, F(\omega), X): C F(\omega)=R(\omega)) \leq 0)$

$=P\left(t^{*}(\omega, X) \leq 0\right)$,

where

$$
t^{*}(\omega, X):=\inf (t(\omega, F(\omega), X): C F(\omega)=R(\omega))
$$

is the minimal value of the program

$\min t(\omega, F(\omega), X)$

s.t.

$\mathbf{C F}(\omega)=\mathbf{R}(\omega)$

being equivalent to the linear program 
minimize $t$

s.t.

$$
\begin{aligned}
& F_{j}-F_{j}^{U}(\omega, X)-t \leq 0, j=1, \ldots, n \\
& F_{j}^{L}(\omega, X)-F_{j}-t \leq 0, j=1, \ldots, n \\
& C F(\omega)=R(\omega)
\end{aligned}
$$

with the variables $F_{1}, F_{2}, \ldots, F_{n}, t$.

Because of condition (19.1), for each $(\omega, X)$ we get

$$
t(\omega, F(\omega), X) \geq \max _{1 \leq j \leq n} \frac{1}{2}\left(F_{j}^{L}(\omega, X)-F_{j}^{U}(\omega, X)\right)>-\infty
$$

for arbitrary $F(\omega)$; hence, the objective function of the linear program (21) is bounded from below for all $(\omega, X)$. Since the LP (21) always has a feasible solution, for each $(\omega, X)$ an optimal solution $\left(F_{t}^{*}\right)$ of $(21)$ is guaranteed, and we have that

$$
t^{*}=t\left(\omega, F^{*}(\omega), X\right)=t^{*}(\omega, X) \text {. }
$$

Consequently, by means of duality theory the optimal value $t^{*}(\omega, X)$ of the equivalent programs (20.2) and (21) can be represented also by the optimal value of the dual program of (21) given by

$$
\begin{aligned}
& \max R(\omega) \cdot u-F^{U}(\omega, X) \cdot \vec{u}^{+}+F^{L}(\omega, X) \cdot \vec{u}^{-} \\
& \text {s.t. } \\
& \begin{array}{c}
C^{\prime} u-\vec{u}^{+}+\vec{v}^{-}=0 \\
1^{\prime} \vec{u}^{+}+1: \vec{u}^{-}=1 \\
\vec{u}^{+} \geq 0, \vec{v}^{-} \geq 0
\end{array}
\end{aligned}
$$

where $u \in \Re^{n}$ is not restricted. Thus, $t^{*}(\omega, X)$ reads

$$
t^{*}(\omega, X)=\max \left(\left(\begin{array}{c}
\mathrm{R}(\omega) \\
-\mathrm{F}^{\mathrm{U}}(\omega, \mathrm{X}) \\
\mathrm{F}^{\mathrm{L}}(\omega, \mathrm{X})
\end{array}\right)^{\prime} \delta:\left(\begin{array}{c}
\mathrm{u} \\
\tilde{u}^{+} \\
\tilde{v}^{-}
\end{array}\right)=: \delta \in \Delta_{0}\right\},
$$

where $\Delta_{0}$ denotes the convex polyhedron in $x^{m+2 n}$ represented by the constraints (23.1)-(23.3) of the LP (23). Taking any subset $\Delta_{1} \subset_{\Delta_{0}}$ of $\Delta_{0}$, and defining then $t_{1}^{*}(\omega, x)$ by

$$
t_{1}^{*}(\omega, X):=\sup \left(\left(\begin{array}{c}
R(\omega) \\
-F^{U}(\omega, X) \\
F^{L}(\omega, X)
\end{array}\right)^{\prime} \delta: \delta \in \Delta_{1}\right),
$$

we get

$$
t^{*}(\omega, x) \geq t_{1}^{*}(\omega, x)
$$

which yields for $P(X)$ the following upper bound:

$$
P(X)=P\left(t^{*}(\omega, X) \leq 0\right) \leq P\left(t_{1}^{*}(\omega, X) \leq 0\right) .
$$




$$
\delta^{(\ell)}=\left(\begin{array}{c}
\mathrm{u}^{(\ell)} \\
\tilde{u}^{+(l)} \\
\tilde{u}^{-(l)}
\end{array}\right), \ell=1, \ldots, \ell_{0},
$$

denote the extreme points of the convex polyhedron $z_{0}$, then

$$
t^{*}(\omega, X)=\max _{1 \leq \ell \leq \ell} R(\omega)^{\prime} u^{(\ell)}-F^{U}(\omega, X) \cdot \tilde{u}^{+(l)}+F^{L}(\omega, X) \cdot \tilde{u}^{-(\ell)},
$$

which shows that $t^{*}(., X)$ is measurable. Hence, $S\left(F^{L}(),. F^{U}().\right)=(\omega \in \Omega$ :

$\left.t^{*}(\omega, X) \leq 0\right)$ is measurable, cf. $(10),(20)$, and we get

$$
P(X)=P\left(R(\omega)^{\prime} u^{(l)}-F^{U}(\omega, X)^{\prime} \tilde{u}^{+(l)}+F^{L}(\omega, X) \cdot \tilde{u}^{-(l)} \leq 0,1 \leq \ell \leq \ell\right) \text {. }
$$

According to $(6),(7)$, the survival, failure, resp., of the underlying structure can be described by the inequality

$t^{*}(\omega, X) \leq 0, t^{*}(\omega, X)>0$, respectively.

Thus, the structure fails if

$$
\begin{array}{r}
\mathrm{R}(\omega)^{\prime} \mathrm{u}^{(\ell)}+\mathrm{F}^{\mathrm{U}}(\omega, \mathrm{X}) \cdot \tilde{\mathrm{u}}^{+(\ell)}-\mathrm{F}^{\mathrm{L}}(\omega, \mathrm{X}) \cdot \tilde{\mathrm{u}}^{-(\ell)}>0 \text { for at } \\
\text { least one } 1 \leq \ell \leq \ell{ }_{0} ;
\end{array}
$$

obviously, (27.1) represents the different failure modes of the structure.

Having a certain number $\ell_{1} \leq \ell_{0}$ of basic solutions $\delta^{\left(\ell_{\tau}\right)}, r=1, \ldots, \ell_{1}$, of the LP (23), and defining

$$
\begin{aligned}
& \tilde{\mathrm{t}}_{1}^{*}(\omega, \mathrm{X}):=\max _{1 \leq r \leq \ell_{1}} \mathrm{R}(\omega)^{\prime} \mathrm{u}^{\left(\ell_{\tau}\right)}-\mathrm{F}^{\mathrm{U}}(\omega, \mathrm{X}) \cdot \tilde{\mathrm{u}}^{+}{ }^{\left(\ell_{\tau}\right)}+\mathrm{F}^{\mathrm{L}}(\omega, \mathrm{X})^{\prime} \tilde{\mathrm{u}}^{-}{ }^{\left(\ell_{\tau}\right)}, \\
& \text { corresponding to }(25.1) \text {, here we get } \\
& \mathrm{t}^{*}(\omega, \mathrm{X}) \geq \tilde{\mathrm{t}}_{1}^{*}(\omega, \mathrm{X})
\end{aligned}
$$

and therefore

$$
P(X)=P\left(t^{*}(\omega, X) \leq 0\right) \leq P\left(\tilde{t}_{1}^{*}(\omega, X) \leq 0\right) .
$$

\section{THE PROBABILITY OF FAILURE $p_{f}$}

According to (8), (10), (20) and (27), for the probability of failure $p_{f}:=1-p_{s}=1-P(X)$ we obtain

$$
\begin{aligned}
p_{f} & =P\left(t^{*}(\omega, X)>0\right) \\
& =P\left(R(\omega) \cdot u-F^{U}(\omega, X) \cdot \tilde{u}^{+}+F^{L}(\omega, X) \cdot \tilde{u}^{-}>0 \text { for at least one }\left(\begin{array}{l}
w \\
\tilde{u}^{+} \\
\tilde{u}^{-}
\end{array}\right) \in \Delta_{0}\right) \\
& =P\left(R(\omega) \cdot u^{(\ell)}-F^{U}(\omega, X) \cdot \tilde{u}^{+(\ell)}+F^{L}(\omega, X) \cdot \tilde{u}^{-(\ell)}>\begin{array}{l}
>\text { for at least } \\
\text { on } 1 \leq \ell \leq \ell
\end{array}\right) \\
& =P\left(\bigcup_{\ell=1}^{\ell} F_{\ell}(X)\right),
\end{aligned}
$$


where $\mathbf{F}_{\ell}(\mathrm{X})$ denotes the $\ell$-th failure domain

$$
\begin{aligned}
\mathbf{F}_{\ell}(\mathrm{X}): & :\left(\omega \in \Omega: R(\omega)^{\prime} \mathrm{u}^{(\ell)}-\mathrm{F}^{\mathrm{U}}(\omega, \mathrm{X}) \cdot \tilde{u}^{+(\ell)}+\mathrm{F}^{\mathrm{L}}(\omega, \mathrm{X}) \cdot \tilde{u}^{-(\ell)}>0\right\} \\
& =\left\{\omega \in \Omega: \mathrm{M}_{\ell}(\omega, \mathrm{X})<0\right\}
\end{aligned}
$$

with the corresponding limit state function

$$
M_{\ell}(\omega, X):=F^{U}(\omega, X) \cdot \tilde{u}^{+(\ell)}-F^{L}(\omega, X)^{\prime} \tilde{u}^{-(\ell)}-R(\omega) \cdot u^{(\ell)},
$$

$\ell-1, \ldots, \ell_{0}$; especially, for trusses, cf. (2.2), (18.1), we find

$$
\mathrm{M}_{\ell}(\omega, \mathrm{X}):=\sigma^{\mathrm{U}}(\omega)^{\prime} \mathrm{A}(\mathrm{X}) \mathrm{d}^{\mathrm{u}^{+(\ell)}}-\sigma^{\mathrm{L}(\omega)^{\prime} \mathrm{A}(\mathrm{X}) \mathrm{d}^{\mathrm{u}^{-(\ell)}}-\mathrm{R}(\omega)^{\prime} \mathrm{u}^{(\ell)}} .
$$

Using known inequalities for probabilities, for $\mathrm{p}_{f}$ we find the bounds

$$
\max _{1 \leq \ell \leq \ell} p_{f, \ell} \leq p_{f} \leq \sum_{\ell=1}^{\ell} p_{f, l},
$$

where $\mathrm{p}_{f, \ell}$ is given by

$$
\begin{aligned}
\mathrm{p}_{\mathrm{f}, \ell}: & =\mathrm{P}\left(\mathrm{F}_{\ell}(\mathrm{X})\right)=\mathrm{P}\left(\mathrm{M}_{\ell}(\omega, \mathrm{X})<0\right) \\
& =\mathrm{P}\left(\mathrm{F}^{\mathrm{U}}(\omega, \mathrm{X}) \cdot \tilde{\mathrm{u}}^{+(\ell)}-\mathrm{F}^{\mathrm{L}}(\omega, \mathrm{X})^{\prime} \tilde{\mathrm{u}}^{-(\ell)}<\mathrm{R}(\omega)^{\prime} \mathrm{u}^{(\ell)}\right. \\
& =1-\mathrm{P}\left(\mathrm{R}(\omega)^{\prime} \mathrm{u}^{(\ell)} \leq \mathrm{F}^{\mathrm{U}}(\omega, \mathrm{X})^{\prime} \tilde{\mathrm{u}}^{+(\ell)}-\mathrm{F}^{\mathrm{L}}(\omega, \mathrm{X})^{\prime} \tilde{\mathrm{u}}^{-(\ell)}\right),
\end{aligned}
$$

and sharper bounds can be obtained by using more genral Bonferroni-inequalities for probabilities.

7 REPRESENTATION OF $\mathrm{p}_{\mathbf{S}}$ BY USING CONES

According to (9) we have that

$$
\mathbf{p}_{\mathbf{S}}=\mathrm{P}\left(\mathrm{R}(\omega) \in \mathrm{C}\left[\mathrm{F}^{\mathrm{L}}(\omega), \mathrm{F}^{\mathrm{U}}(\omega)\right]\right),
$$

where $\left[\mathrm{F}^{\mathrm{L}}, \mathrm{F}^{\mathrm{U}}\right]$ is given by (8.2). Representing therefore the vector $\mathrm{F}$ of internal member forces/bending-moments by

$$
F=F^{L}+\Delta F^{L}=F^{U}-\Delta F^{U}
$$

with $n$-vectors $\Delta F^{U}, \Delta F^{L} \geq 0$, the condition $R \in C\left[F^{L}, F^{U}\right]$ can be represented by

$$
\begin{aligned}
& R-C F^{U}=-C \Delta F^{U} \\
& -\left(F^{U}-F^{L}\right)=-A F^{U}-\Delta F^{L} \\
& \Delta F^{U} \geq 0, \Delta F^{L} \geq 0 .
\end{aligned}
$$

Thus, we consider the cone $Y_{0} \Re^{m+n}$ defined by

$$
\begin{aligned}
& Y_{0}=\left(\left(\begin{array}{ll}
C & 0 \\
I & I
\end{array}\right)\left(\begin{array}{c}
\Delta F^{U} \\
A F^{L}
\end{array}\right): \Delta F^{U} \geq 0, \Delta F^{L} \geq 01\right. \\
& =\left\{\sum_{k=1}^{2 n} \alpha_{k} y_{k}: \alpha_{k} \geq 0, k=1, \ldots, 2 n\right) \text {, }
\end{aligned}
$$


where the cone-generators $y^{(k)}, k=1, \ldots, 2 n$, are given by

$$
y^{(k)}:=\left(\begin{array}{l}
c_{k} \\
e_{k}
\end{array}\right), 1 \leq k \leq n, y^{(k)}:=\left(\begin{array}{l}
0 \\
e_{k}
\end{array}\right), n<k \leq 2 n,
$$

and $c_{k}, e_{k}$ denotes the $k$-th column of $c$, of the $n \times n$ identity matrix $I$, respectively. Having $Y_{0}$, the set $S\left(F^{L}(),. F^{U}().\right)$ defined in (8.1) can be described by

$$
S\left(F^{L}(.), F^{\mathrm{U}}(.)\right)=\left(\omega \in \Omega:\left(\begin{array}{l}
\mathrm{R}(\omega)-\mathrm{CF}^{\mathrm{U}}(\omega, \mathrm{X}) \\
-\mathrm{F}^{\mathrm{U}}(\omega, \mathrm{X})+\mathrm{F}^{\mathrm{L}}(\omega, \mathrm{X})
\end{array}\right) \in(-1) \mathrm{Y}_{\mathrm{o}}\right\},
$$

which shows again the measurability of $S\left(F^{L}(),. F^{U}().\right)$. Moreover, the probability function $\mathrm{P}=\mathrm{P}(\lambda, \mathrm{X})$ representing $(10)-(10.3)$ can be given by

$$
P(\lambda, X)=P\left(\left(\begin{array}{l}
C F^{U}(\omega, X)-\lambda R_{0}(\omega) \\
F^{U}(\omega, X)-F^{L}(\omega, X)
\end{array}\right) \in Y_{0}\right) \text {. }
$$

According to the representation (32) of $\mathrm{Y}_{0}$, there are a finite number of boundary hyperplanes in $x^{\mathrm{m}+\mathrm{n}}$, represented by vectors $\eta^{(\ell)}-\left(\begin{array}{c}\mathrm{w}^{(\ell)} \\ \mathrm{v}^{(\ell)}\end{array}\right), \ell=1, \ldots, \ell_{0}^{\prime}$, such that

$$
\mathrm{x}_{0}=\left\{\mathrm{y}=\left(\mathrm{v}_{\mathrm{v}}^{\mathrm{w}}\right) \in \mathrm{R}^{\mathrm{m}+\mathrm{n}}: \mathrm{y}^{\prime} \eta^{(\ell)}=\mathrm{w}^{\prime} \mathrm{w}^{(\ell)}+\mathrm{v}^{\prime} \mathrm{v}^{(\ell)} \geq 0, \quad 1 \leq \ell \leq \ell_{0}^{\prime}\right\} \text {. }
$$

Hence, because of (33) and (34), the survival of the structure can be represented also by the inequalities

$$
\left(R(\omega)-C^{U}(\omega, X)\right)^{\prime} w^{(l)}+\left(-F^{U}(\omega, X)+F^{L}(\omega, X)^{\prime} v^{(l)} \leq 0,1 \leq l \leq l\right. \text { ', }
$$

which yields

$$
\left(R(w)^{\prime} w^{(l)}-F^{U}(w, X)^{\prime}\left(C^{\prime} w^{(\ell)}+v^{(l)}\right)+F^{L}(w, X)^{\prime} v^{(l)} \leq 0,1 \leq \ell \leq \ell{ }_{0}^{\prime},\right.
$$

and therefore

$$
P_{s}=P\left(R(\omega)^{\prime} w^{(l)}-F^{U}(\omega, X)^{\prime}\left(C^{\prime} w^{(\ell)}\right)+v^{(l)}+F^{L}(\omega, X)^{\prime} v^{(l)} \leq 0, \quad 1 \leq l \leq \ell_{0}^{\prime}\right) .
$$

Obviously, the conditions for structural safety given by (27) and (35) coincide.

For arbitrary subset $\mathrm{Y}_{0}^{(\ell)}, \ell=1,2$, such that

$$
\mathrm{Y}_{0}^{(1)} \subset \mathrm{Y}_{0} \mathrm{CY}_{\mathrm{o}}^{(2)} \text {, }
$$

we get

$$
\mathrm{p}_{\mathrm{s}}^{(1)} \leq \mathrm{p}_{\mathrm{s}} \leq \mathrm{p}_{\mathrm{s}}^{(2)}
$$

where the bounds $\mathrm{p}_{s}^{(\ell)}, \ell=1,2$, are defined by

$$
\mathbf{P}_{S}^{(\ell)}:=P\left(\left(\begin{array}{l}
\mathrm{CF}^{\mathrm{U}}(\omega, \mathrm{X})-\mathrm{R}(\omega) \\
\mathrm{F}^{\mathrm{U}}(\omega, \mathrm{X})-\mathrm{F}^{\mathrm{L}}(\omega, \mathrm{X})
\end{array}\right) \in \mathrm{Y}_{\mathrm{O}}^{(\ell)}\right), \ell=1,2 .
$$




\subsection{Construction of approximating cones}

Approximations $\mathrm{Y}_{0}^{(l)}, \ell=1,2$, of $\mathrm{Y}_{0}$ can be obtained by spherical or ellipsoidal approximation of the convex polyhedron generated by $y^{(k)}, k=1, \ldots, 2 n$.

8 COMPUTATION OF SENSITIVITIES OF PROBABILITIES OF SURVIVAL AND PROBABILITIES OF FAILURE

Sensitivities of $p_{s}, p_{f}$ with respect to the load factor $\lambda$ and the vector $X$ of design variables $x_{k}, k=1,2, \ldots, r$, can be obtained now by applying the differerentiation formulas given in [6], [7], [8], [9] to the probability functions $(10),(10.2)-(10.5),(16.1),(18.1),(26.1),(33.1)$.

\section{REFERENCES}

[1] Arnbjerg-Nielsen, Torben: Rigid-ideal plastic model as a reliability analysis tool for ductile structures. Ph.D. Dissertation, Technical University of Denmark, Lyngby 1991

[2] Augusti, G. et al.: Probabilistic Methods in Structural Engineering. Chapman and Ha11, London-New York 1984

[3] Haftka, R.T. et a1.: Elements of Structural Optimization. Kluwer, Dordrecht-Boston-London 1990

[4] Hodge, P.G.: Plastic Analysis of Structures. Mc Graw-Hill, New York 1959

[5] Kirsch, U.: Structural Optimization. Springer-Verlag, Berlin-HeidelbergNew York 1993

[6] Marti, K.: Differentiation of Probability Functions: The Transformation Method. To appear in "Computers and Mathematics with Applications", 1995

[7] Marti, K.: Differentiation Formulas for Probability Functions: The Transformation Method. To appear in "Mathematical Programming, Series $B^{\prime \prime}, 1995$

[8] Marti, K.: Differentiation of Probability Functions Arising in Structural Reliability. In: R. Rackwitz (ed.): Reliability and Optimization of Structural Systems. Chapman and Hall, London 1995

[9] Marti, K.: Computation of Probability Functions and its Derivatives by means of Orthogonal Functions Series Expansions. In: K. Marti, P. Kal1 (eds.): Stoch. Programming: numerical techniques and engineering applications. LNEMS Vol. 423, Springer-Verlag, Berlin-Heidelberg-New York 1995

[10] Nafday, A.M., Corotis, R.B.: Failure Mode Enumeration for System Reliability Assessment by Optimization Algorithms. In: P. Thoft-Christensen (ed.): Reliability and Optimization of Structural Systems. SpringerVerlag, Berlin-Heidelberg-New York 1987 